\title{
Development of a General Purpose DSMC Flow Solver on Unstructured Meshes
}

\author{
V. Tenishev*, M. Combi ${ }^{\dagger}$ \\ Space Physics Research Laboratory, Department of Atmospheric, Oceanic and Space Sciences, University \\ of Michigan, Ann Arbor, MI
}

\begin{abstract}
A general purpose kinetic parallel flow solver was developed based on DSMC methodology. Basic characteristics of the solver and some examples of its application are outlined in the paper.
\end{abstract}

\section{Introduction}

One of the most important aspects of the rarefied gas simulation is a high value of the Knudsen number $K n=\lambda / L$ in the flow and, consequently, the requirement of solution of the Boltzmann equation

$$
\frac{\partial f_{i}}{\partial t}+\mathbf{v}_{i} \nabla f_{i}+\frac{\mathbf{a}_{i}}{m_{i}} \nabla_{v} f_{i}=\left(\frac{\delta f_{i}}{\delta t}\right)_{c o l l}
$$

The situation is usually more complicated because most of the practical applications involve consideration of diatomic gases, which can be excited vibrationally and rotationally and for which ionization, dissociation and chemical transformations are possible. A need to model non-equilibrium hypersonic flows motivated the development ${ }^{1}$ of numerical techniques to treat such problems. Nowadays the DSMC method ${ }^{2}$ is de facto the standard method for rarefied gas dynamics, where the state of the rarefied gas flow, which is presented by an assembly of molecules, is determined by collisional dynamics of a finite number of model particles and, hence, hold potential for providing information on flows where the collisional rate is not sufficient to maintain equilibrium energy distribution.

\footnotetext{
*Research Assistant; vtenishe@umich.edu

${ }^{\dagger}$ Senior Research Scientist
}

This paper presents the current status of development of a general purpose DSMC flow solver and some results of its application.

\section{Code}

Current problems of gas dynamics cannot be solved by methods other than numerical ones. A wide range of practically important problems requires developing a highly general numerical tool. $\mathrm{C}++$ language was chosen in the present work.

\section{Requirements}

The following main principles guided the development of the code:

1. Portability: the code was tested on a set of different platforms and compilers.

2. Easy modification of the core of the code: change of the basic physicochemical models installed into the code should not require any changes in any other part of the code.

3. Careful usage of resources: special attention has been paid to optimize allocation of memory and CPU resources.

4. Parallel implementation: MPI library has been used in parallelization of the system. Dynamic and static load balancing capabilities were implemented.

5. Easy adaptation of the code to particular physical problem (without modifacation of the core of the code).

6. Friendly user interface: easily understandable input and output files.

7. Pre/post-processing: including pre/postprocessor into the core of the system, 
allow running and post-processing on different platforms.

8. Optimization of the numerical procedure for the current problem: change main parameters of simulation on order to improve overall performance.

\section{Basic Specification of the System}

The code potentially can be applied to a wide range of problems. The following is the list of its main features.

1. Neutral gas flow analysis in 1, 2, 3D cases. Spherical- and axis-symmetry can be taken into account.

2. The flow field is reconstructed on an unstructured mesh.

3. Multiple species gas flows can be modeled. Chemical reactions and relaxation of internal degrees of freedom can be considered.

4. A set of molecular and collisional models is available.

5. Mean free path and model particle weight adaptation has been implemented

\section{Code Adaptation for Particular Problem}

The core contains a general set of models (molecular models, collisional, chemical models). Some cases require introduction of additional particles or/and physicochemical models to solve particular problem. Such models may contain a set of approximations or/and experimental data specifically suited for the problem and, so, they may not be useful for any other cases. It is good to have the possibility to incorporate models into numerical procedure without any modification of the core of system. There are several difficulties that have to be solved in this way. Based on capabilities that are provided by $\mathrm{C}++$, an approach of treating problem-dependent models was developed and incorporated into the system.

\section{Optimization techniques}

The accuracy of the numerical results, which are obtained by the DSMC method, is very dependent on the size of the sample and, finely, on the number of model particles per cell. So, in order to make the most effective use of computer resources, it is desirable to get the distribution of model particles over the domain close to uniform. This problem is especially important for the case of a 2D axisymmetrical simulation. There are several methods ${ }^{2}$ to control the distribution of particles: variation of time steps, grid manipulation and direct variation of a particle's weight.

Several techniques could be used to decrease total computational cost of steady state simulation. Due to methodology of the DSMC method, a steady state solution can be obtained as the limit of unsteady flow simulation, where convergence takes a considerable part of total computation time. To get faster convergence, it is possible, starting with low accuracy, to improve it gradually as the current solution converges to the finial result. One of the possible implementations ${ }^{3}$ of the strategy is to gradually increase total number of model particles as the flow pattern converges to the steady state. Another scheme was developed and implemented in the current work. The simulation is conducted on a set of meshes with different levels of refinement, starting with a coarse grid at the very beginning and maintaining the number of model particles per cell in desired limits. As the number of collisional cells and particles employed increase, the resolution of the computational procedure improves both in terms of flow macroparameters distribution and captured physical processes.

The use of parallel computers can significantly increase the range of application of the DSMC method. Effective use ${ }^{4}$ of such computational systems can be achieved only with careful load balancing.

Static and dynamic load balancing capabilities are incorporated into the current implementation. Different criteria are available for the balancing. Static load balancing may be based either entirely on parameters of initial flow condition or on some knowledge about the structure of converged solution. In the first case, the load is balanced by equipartitioning of total number of particles, total volume or total cell number among all available processors. In addition to them, a total execution time can be used as a criterion in dynamic load balancing. 


\section{Applications of the code}

\section{Cometary Comae Simulation: Generic Jupiter Family Comet}

The cometary atmosphere is a unique phenomenon in the solar system. It represents highly extended dusty gas cloud with a large range of change of its macroparameters from fluid to collisionless. So, kinetic methods are the most suitable tools for simulation processes in cometary comae.

\section{PHYSICAL MODEL}

When the nucleus approaches the sun, it absorbs solar radiation, resulting in an increase of gas sublimation from the surface. Because the gravitational force is negligible, the gases leaving the surface form a cometary exosphere. The radiation reaching the surface and supplying energy for sublimation is partially adsorbed dusty atmosphere. Any changes in the gas and dust production result in changes of optical characteristics of the cloud. The main volatile component of the cometary coma is water. So, water sublimation controls sublimation of others components when a comet is within about 4 astronomical units (AU) of the sun. Freshly evaporated molecules are photodissociated and photoionized, and therefore most of the chemical kinetics of cometary atmospheres involves the resulting highly reacting ions and radicals ${ }^{5}$. Most of the absorption of solar radiation takes place at the surface or in the dense region near the surface. Models of temperature and gas production distributions on the surface of a spherical nucleus can be found ${ }^{6,7}$. The simplest model of gas production assumes an optically thin coma, which is an excellent approximation for most comets, most of the time.

The conditions for a comet coma usually consider the gas at the surfaces to be stationary at a given temperature. This assumption neglects the real physics of the gas surface interface. But to get correct structure of the flow field in the near nucleus region, it is more important that the flux integrated around the surface equals the desired production rate and the gas temperature near the surface is close to vaporization temperature of water. Due to absence of gravity, the downward flux is negligible. This result in upward-half Maxwellian distribution of water vapor in a gas layer where thermodynamic equilibrium is not held. So, in a cometary atmosphere, unlike that of planets atmospheres, the mean flow velocity can be comparable with to the thermal speed ${ }^{5}$, therefore methods adopted for studying planetary atmospheres are not longer valid. The flow then quickly thermalizes and passes smoothly through the sonic transition.

Two-dimensional axisymmetrical models for six species $\left(\mathrm{H}_{2} \mathrm{O}, \mathrm{CO}, \mathrm{OH}, \mathrm{H}_{2}, \mathrm{O}, \mathrm{H}\right)$ were considered in this work. A hard sphere collision model was assumed for intermolecular collisions. For water-water collisions the viscosity equivalent cross section ${ }^{8}$ has been used. For other collision pairs, collision cross sections based on relevant atomic data were used ${ }^{9}$.

The cometary nucleus was assumed to be spherical with a given radius and axisymmetrical gas emission. The gas flux from the dayside is controlled by absorption of solar radiation, and the flux is varying as the square of the cosine of the subsolar angle. It was assumed that only two gaseous species sublimate from the surface: $\mathrm{H}_{2} \mathrm{O}$ and $\mathrm{CO}$. Both species are in equilibrium with the surface and in the outflow mixture is partitioned on $80 \%$ for $\mathrm{H}_{2} \mathrm{O}$ and $20 \%$ for $\mathrm{CO}$.

Photochemistry is included into the model. The main reaction branches are presented in Table 1

\begin{tabular}{|l|l|}
\hline Reaction & $\begin{array}{l}\text { Branching } \\
\text { ratio }\end{array}$ \\
\hline $\mathrm{H}_{2} \mathrm{O} \rightarrow \mathrm{H}+\mathrm{OH}$ & 0.88 \\
\hline $\mathrm{H}_{2} \mathrm{O} \rightarrow \mathrm{H}_{2}+\mathrm{O}$ & 0.22 \\
\hline $\mathrm{OH} \rightarrow \mathrm{O}+\mathrm{H}$ & 1.00 \\
\hline $\mathrm{H}_{2} \rightarrow \mathrm{H}+\mathrm{H}$ & 1.00 \\
\hline
\end{tabular}

Table 1 Photochemical branching

\section{STATEMENT OF THE PROBLEM}

A comet with a production rate of $3 \times 10^{28}$ molecules/sec was chosen, as typical of Jupiter family comets which will be spacecraft mission targets over the next decade or two. The radius of the nucleus was taken to be $10 \mathrm{~km}$ and the gas production rate was distributed as the square of the cosine of the subsolar angle with no gas emission on the night side. The gas was initiated at the surface at rest and with a temperature of $180 \mathrm{~K}$. The results of the calculation are shown in Fig. 1,2.

For photochemical reactions, the rates are typically much smaller than collision rates near the nucleus. Taking the characteristic time 
constant for particular photochemical reaction $\tau_{i}$ the probability of photodecay for a single molecule at a given time step $\Delta t$ is

$$
p_{i}=1-\exp \left(-\Delta t / \tau_{i}\right)
$$

The regime of expansion of the cometary coma changes from collision dominated near the surface of the nucleus though transitional one to collisionless in the far field. Consequently, all of the regimes have to be described by numerical approach chosen for simulation, which makes questionable applicability of fluid based approximations.

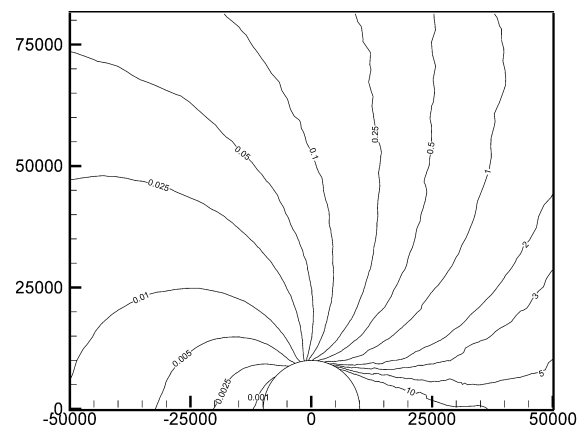

Fig. 1 The two-dimensional field of Knudsen numbers for the case of a generic Jupiter family comet.

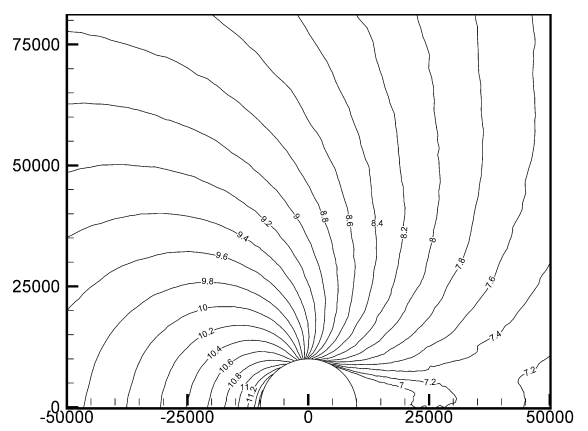

Fig. 2 The two-dimensional field of decimal logarithm of water density (in $\mathrm{cm}^{-3}$ ) for the case of a generic Jupiter family comet.

\section{Gas/Dust Interaction in cometary comae}

Our present understanding of cometary nucleus is based on idea of "dirty snowball" according to which the nucleus consists of frozen volatiles and nonvolatile dust. The sublimated gas molecules drag away dust particles from the surface of cometary nuclei, so, the gas/dust interaction may a have significant effect on macroparameter distributions in comeraty comae. In this work, our particular interest was concentrated on modeling of inner comae processes, the region where dust and gas are produced and accelerated to their terminal velocities.

The following mechanism of dust particle acceleration is adopted here. It is assumed that an influence of gravitational force is negligible and, so, dust particles acceleration is only due to collisions with the gas molecules

Different kinds of gas molecules/dust surface interaction mechanisms can be easily introduced into the model, the simplest version of which assumes elastic reflection on the surface of the dust particle. In the current work, its simplified approximation has been used. Taking $a$ as the dust particle radius, $V_{d u s t}$ and $V_{m o l}$ as dust and gas particle's velocities, the change in momentum of the dust particle during time interval $\Delta t$ can be expressed as

$\Delta M_{\text {dust }}=$

$=\Delta t \sum_{m o l} 2 \pi a^{2} m_{m o l}\left|V_{m o l}-V_{d u s t}\right|\left(V_{m o l}-V_{d u s t}\right) \frac{F_{m o l}}{V_{c e l l}}$

Here, $F_{m o l}$ is the model particle weight of gas molecule and $V_{\text {cell }}$ the volume of the computational cell. The model has been validated via comparison with test solution obtained by a fluid/dust model ${ }^{5}$. For the problems of cometary comae simulation, due to a wide range of collisional regimes in the flow, application of the fluid approximation is restricted.

The problem was considered in 1D spherically symmetrical geometry. The nucleus radius $R=5.3 \mathrm{~km}$ and gas production rate of $3.5 \times 10^{28}$ molecules/s corresponds to parameters of comet Borrelly at heliocentric distance of $1.36 \mathrm{AU}$. It was assumed that only $\mathrm{H}_{2} \mathrm{O}$ molecules are sublimates from the surface. The dust/gas mass ratio is 0.4 .

Some obtained results are shown in Fig. 3,4 . 


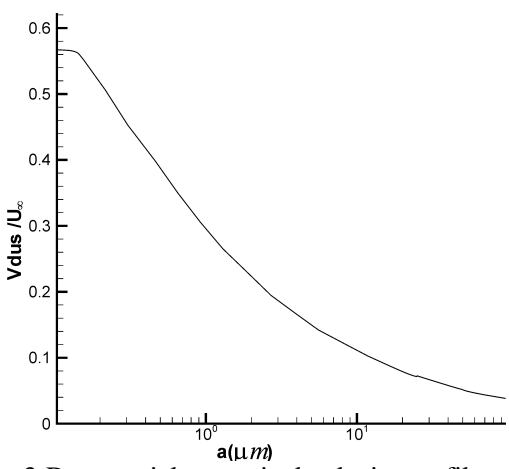

Fig. 3 Dust particles terminal velocity profile as the function of its radius. Here $U_{\infty}$ is the terminal velocity of the gas phase.

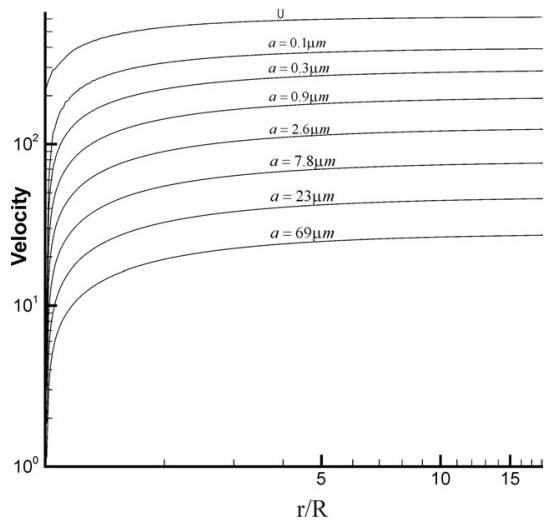

Fig. 4 Radial mean velocity $(\mathrm{m} / \mathrm{s})$ profiles for dust particles of a given size and the gas phase in the cometary coma as a function of a distance from the nucleus.

\section{D Two Body Interaction Problems}

The ultimate goal of the work is developing of a general purpose DSMC flow solver that can be used to solve a wide range of problems in complex geometries. A two-body interaction problem has been chosen to demonstrate the capability of the code to treat complex 3D flow problems. The geometry of the problem is presented on the Fig. 5.

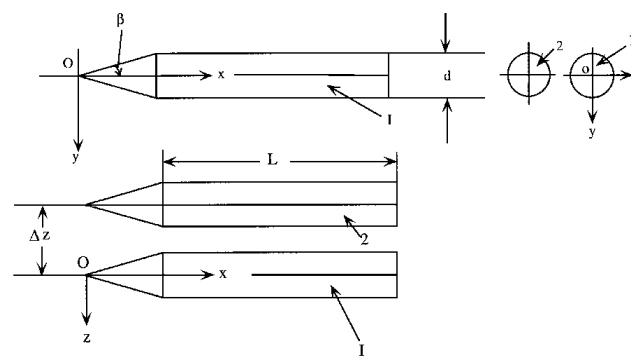

Fig. 5 Geometry sketch for the 3D two body interaction problem

Two bodies of revolution are cone/cylinder combination. The cone semi-angle is $\beta=20^{\circ}$, the cylinder length is $L=250 \mathrm{~mm}$, its diameter is $d=50 \mathrm{~mm}$. The distance between the body axes is $\Delta z=70 \mathrm{~mm}$. The bodies of revolution were aligned parallel, without relative displacement at a zero incidence angle.

The flow of $\mathrm{N}_{2}$ has been simulated on a mesh with $5.7 \times 10^{6}$ computational cells using $\sim 70 \times 10^{6}$ model particles. The upstream conditions are, $\quad U_{\infty}=1242.6 \mathrm{~m} / \mathrm{s}$, $T_{\infty}=51 K, \quad n_{\infty}=8.95 \times 10^{21}, \quad$ which corresponds to Mach number of $M=9.91$. Some of the results are presents of Fig. 6, 7 .

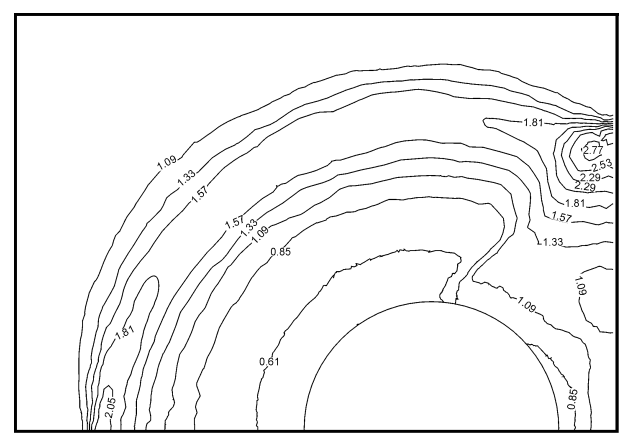

Fig.6 Normalized number density profile at a distance of $0.67 \mathrm{~L}$ from the leading edge of the body 


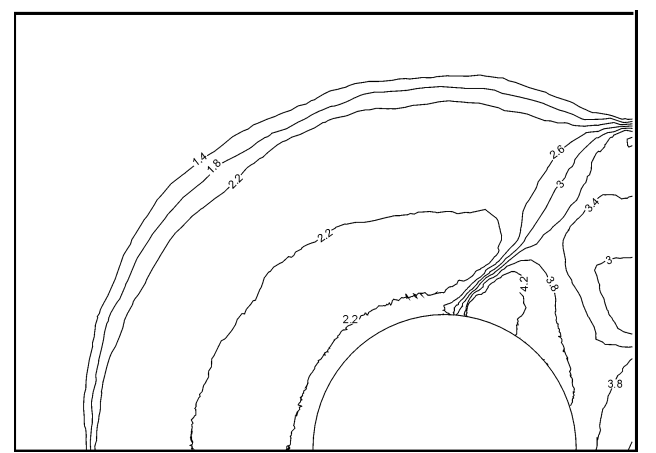

Fig. 7 Normalized temperature profile at a distance of $0.67 \mathrm{~L}$ from the leading edge of the body

\section{Conclusion}

The current state of developing a general-purpose kinetic flow solver was presented. The tool has been validated via comparison with solutions of a set of test problems.

Main features of the code were outlined. It was stressed that portability, modularity, easy adaptation to particular problem, parallel implementation and friendly user interface makes the developed code a useful tool for analysis of complex multidimensional gas flows of chemically reactive mixtures. Discretization of the flow field on unstructured meshes allows the code to be used for solution of problems of internal and external gas flow in the case of complex geometries. Adaptation capabilities can be utilized to optimize overall performance.

In the paper, some examples of application of the code have been presented. A problem of two-phase dust/gas flow has been considered.

\section{References}

1. G. Bird, Molecular Gas Dynamics and the Direct Simulation Monte Carlo Method, Clarendon Press, 1994

2. G.N. Markelov, A.N. Kudryavtsev, M.S. Ivanov, Continuum and Kinetic Simulation of Shock Wave/Laminar Boundary Layer Interaction In Hypersonic Flows, AIAA99-3527

3. M. Ivanov, G. Markelov, S. Gimelshein, Statistical Simulation of Reactive Rarefied
Flows: Numerical Approach and Applications, AIAA 98-2669

4. S. Dietrich, I. Boyd, Scalar and Parallel Optimized Implementation of the Direct Simulation Monte Carlo Method, J. Comp. Physics, 126(2):328-342, 1996

5. T. Gombosi, A. Nagy, T. Cravens, Dust and Neutral Gas Modeling of the Inner Atmospheres of Comas, Rev. of Geophysics, Vol. 24, No. 3, 1086

6. A. Enzian, J. Klinger, G. Schwehm, P. Weissman, Temperature and Gas Production Distributions on the Surface of a Spherical Model Comet Nucleus in the Orbit of 46P/Wirtanen, Icarus 138, 74-84 (1999)

7. P. Gutiérrez, J. Ortiz, R. Rodrigo, J. LópezMoreno, A study of water production and temperatures of rotating irregularly shaped cometary nuclei, Astron. Astrophys. 355, 809817 (2000)

8. J. Crifo, Inferences concerning water vapor viscosity and mean free path at low temperatures, Astron. Astrophys. 223, 365368(1989)

9. M. Combi, Time Dependent Gas Kinetics in Tenuous Planetary Atmospheres: The Cometary Coma, Icarus, 1236 207-226 (1996) 